ISSN: 1021-7444

\title{
DINAMICA POBLACIONAL DE Panicum polygonatum Y Axonopus micay BAJO COMBATE CON HERBICIDAS Y CHAPEA, EN UNA PLANTACIÓN BANANERA ${ }^{1}$
}

\author{
Ana María Rodríguez-Ruiz², Renán Agüero-Alvarado3
}

\begin{abstract}
RESUMEN
Dinámica poblacional de Panicum polygonatum y Axonopus micay bajo combate con herbicidas y chapea, en una plantación bananera. El objetivo de esta investigación fue determinar los cambios poblacionales de arvenses poáceas bajo combate químico y de chapea. El trabajo se realizó en una finca bananera en la zona de Cariari de Pococí, entre octubre del 2003 a setiembre 2005. Se estableció un experimento con un diseño de bloques al azar, con cuatro tratamientos: chapeas bimensuales, aplicaciones calendarizadas con glifosato, aplicaciones calendarizadas con glufosinato y tratamiento monitoreado aplicado con base al monitoreo de las arvenses presentes. Las principales poáceas registradas fueron Panicum polygonatum y Axonopus micay. Se realizaron recuentos de las arvenses cada dos meses. A partir de la segunda evaluación el glifosato tendió a disminuir de manera significativa las poblaciones de $P$. polygonatum, en tanto que la chapea favoreció el desarrollo de la misma; con los tratamientos de glufosinato y el monitoreado las poblaciones de la maleza se mantuvieron intermedias. Los tratamientos de glufosinato y la chapea aumentaron las poblaciones de A. micay.
\end{abstract}

Palabras clave: Panicum polygonatum, Axonopus micay, glifosato, glufosinato de amonio, chapea.

\begin{abstract}
Population dynamics of Panicum polygonatum and Axonopus micay in a banana plantation. This study was conducted to assess the population dynamics of two major weeds of the family Poaceae associated with a banana agroecosystem. The experiment was conducted in the area of Cariari de Pococí between October 2003 to September 2005. An experiment with four weed control treatments was established as a randomized blocks design. The treatments were: bimonthly mechanical control with machete, scheduled applications of glyphosate, scheduled applications of gluphosinate, and a treatment based on the monitoring of the weed population. The main Poaceae registered were Panicum polygonatum and Axonopus micay. Weeds were counted every two months. The second evaluation showed that glyphosate tended to reduce significantly the populations of $P$. polygonatum, while mechanical control favored the development of this species; gluphosinate applications resulted in intermediate populations of the weed. Gluphosinate treatments and mechanical control resulted in higher populations of A. micay.
\end{abstract}

Key words: $P$. polygonatum, A. micay, glyphosate, gluphosinate ammonium, mechanical control.

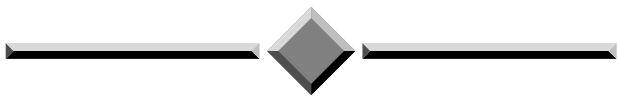

\footnotetext{
Recibido: 17 setiembre, 2010. Aceptado: 23 de mayo, 2011. Esta investigación formó parte del Proyecto "Hacia un manejo racional de la flora vascular asociada al banano", inscrito en la Vicerrectoría de Investigación.

2 Estación Experimental Fabio Baudrit M (EEFBM)-Programa de Manejo Integrado de Malezas. Universidad de Costa Rica (UCR). San José, Costa Rica. amrodriguezster@gmail.com

3 Laboratorio de Arvenses (LAR), Centro de Investigación de Protección de cultivos (CIPROC). Universidad de Costa Rica. San José, Costa Rica.ragueroster@gmail.com
} 


\section{INTRODUCCIÓN}

La dinámica de poblaciones de malezas se refiere al cambio en la composición de plantas en función del tiempo y de las estrategias de manejo. Estos cambios se relacionan con el número de especies y la abundancia de cada una de ellas en un determinado agroecosistema (Young y Evans 1976 y Krebs 1985).

El aumento en la diversidad de especies en un cultivo como banano, se encuentra relacionado con el hecho de encontrarse el mismo como un monocultivo por un largo periodo (Acosta y Agüero 2002 c). Por otra parte, los requerimientos ecológicos del cultivo, de temperaturas elevadas, altas precipitaciones así como la necesidad de suelos fértiles y profundos, crean un ambiente propicio para el desarrollo y establecimiento de muchas arvenses (Gamboa 1983).

Las especies de malezas presentes en el cultivo de banano dependerán de si la plantación se originó a partir de bosque, charrales o potreros; en este último caso el problema de poáceas se vuelve muy agudo y con el tiempo se van seleccionando especies como Paspalum fasciculatum, Paspalum conjugatum, Cynodon dactylum, entre otras (Soto et al. 1992).

Acosta y Agüero (2001) mencionan a Panicum polygonatum (citada como Panicum zizanoides) como una de las principales malezas en el trópico húmedo de Costa Rica; también indican que es una especie que puede presentar densidades promedio de 79 individuos $/ \mathrm{m}^{2}$ (Acosta y Agüero 2002 b). P. polygonatum posee culmos decumbentes que pueden alcanzar una longitud entre 25 a $110 \mathrm{~cm}$, y tienen la capacidad de enraizar en los nudos (Pohl 1980); formando nuevos y numerosos individuos. Por su parte, las especies del género Axonopus agrupan tanto plantas anuales como perennes que se caracterizan por ser cespitosas o rizomatosas y algunas de ellas son pastos estoloníferos (Pohl 1980), característica que favorece su reproducción asexual.

El manejo de las diferentes malezas presentes en el cultivo del banano incluyendo las pertenecientes a la familia Poaceae es indispensable, si se quiere mantener la calidad de la fruta y los buenos rendimientos del cultivo. Sin embargo, a la fecha no se cuenta con esquemas de manejo sustentables, por lo que las malezas siguen interfiriendo con el cultivo (Acosta y Agüero 2002 a).
Por otra parte, en el control de malezas es importante considerar todos aquellos tratamientos que combinen adecuadas prácticas culturales, mecánicas, manuales y el uso racional de herbicidas, encaminados a reducir al mínimo la interferencia y otros efectos negativos que estas plantas ejercen sobre el cultivo y las labores agrícolas (Pinilla y García 2002).

Para el manejo de malezas poáceas en banano se utilizan entre otros los herbicidas glifosato y glufosinato; el primero de ellos un herbicida de acción sistémica, muy móvil dentro de las plantas y de amplio espectro cuando se aplica en posemergencia ya que no sólo controla este tipo de especies sino también muchas otras de hoja ancha; el segundo es un producto de contacto que actúa sobre el tejido verde que llega a tocar y su movilización dentro de la planta es poca; sin embargo, posee un amplio espectro de control de poáceas anuales, perennes y malezas de hoja ancha (Agüero y Rojas 1996, Pitty y Muñoz 1991, Rijn 2000 y Vencill 2002).

El objetivo de este trabajo fue determinar los cambios poblacionales de arvenses poáceas comunes bajo combate químico y de chapea en el cultivo de banano.

\section{MATERIALES Y MÉTODOS}

\section{Ubicación}

Este estudio se desarrolló en la finca bananera Taciafa, situada en Cariari, Pococí, de la provincia de Limón, Costa Rica. El mismo se realizó durante un periodo aproximado de dos años (2003-2005). Según la clasificación de zonas de vida de Holdridge (1979) el lugar corresponde a la formación de bosque tropical húmedo, con una precipitación anual que fluctúa entre los 3500 a $4000 \mathrm{~mm}$. La temperatura anual promedio es de $25^{\circ} \mathrm{C}$ y la humedad promedio del $85 \%$ (Herrera 1985).

\section{Metodología}

El estudio se ubicó en una plantación sembrada con la variedad Gran Enano, en un área aproximada de $7200 \mathrm{~m}^{2}$. Se establecieron cuatro tratamientos de manejo de arvenses en un diseño de bloques completos al azar con cuatro repeticiones. Cada unidad 
experimental contó con área promedio de $400 \mathrm{~m}^{2} \mathrm{y}$ en cada una de ellas se definieron aleatoriamente al inicio, tres puntos de observación entre las hileras, constituyendo un total de 48 puntos de observación. A partir de la aleoterización inicial, estos puntos se mantuvieron como estaciones fijas de muestreo, para las evaluaciones subsiguientes, los mismos se identificaron con estacas. Se evaluaron los siguientes tratamientos: $\mathrm{T}_{1}=$ control con chapeas bimensuales; $\mathrm{T}_{2}=$ control con glifosato cada dos meses; $\mathrm{T}_{3}=$ control con glufosinato cada dos meses; $\mathrm{T}_{4}=$ control monitoreado con chapea, glifosato o glufosinato cada dos meses según la evaluación de la flora presente realizada el día antes de hacer la aspersión.

Para las aplicaciones de los herbicidas se utilizaron bombas de espalda de 16 litros con boquillas de abanico plano 8002. Las dosis de los productos aplicados fueron establecidas con base en las recomendaciones emitidas por las casas comerciales representantes de cada uno de ellos, a saber: glufosinato a 22,5 g i.a. (ingrediente activo) /16 litros de agua y glifosato a $75 \mathrm{~g}$ i.a./16 litros de agua. El volumen de aplicación varió entre los 133 y los 175 litros/ha dependiendo del estado de las malezas. La chapea se realizó con un machete de $80 \mathrm{~cm}$ de longitud.

\section{Cuantificación de la vegetación predominante}

Se realizaron recuentos del número de individuos de las diferentes poáceas presentes en los puntos de muestreo que se definieron entre las hileras del banano, estos recuentos se hicieron antes del inicio de los tratamientos y cada dos meses durante aproximadamente dos años después del primer muestreo. Para tal efecto se utilizó un marco de madera de un metro cuadrado dividido en segmentos de $10 \mathrm{~cm}^{2}$ de área cada uno. El marco se colocó en cada estación de muestreo ubicado en los espacios libres entre las unidades de producción del cultivo. Una vez identificadas las especies, se procedió a realizar el respectivo conteo de individuos en cada sitio.

Los datos se analizaron estadísticamente mediante el programa ANAWIN, según el diseño establecido. El análisis de variación se realizó primero en forma separada para las dos especies de poáceas predominantes. Posteriormente se compararon las poblaciones de ambas especies en cada uno de los tratamientos. Las comparaciones de medias se realizaron mediante la prueba de Tukey al 5\% de probabilidad.

\section{RESULTADOS Y DISCUSIÓN}

Dinámica poblacional por especie

\section{Panicum polygonatum}

$\mathrm{Al}$ inicio todos los tratamientos contaron con poblaciones similares de P. polygonatum (Figura 1). Dos meses después de la primera aplicación, el glifosato mostró una disminución de la maleza en tanto que el glufosinato y el monitoreado presentaron poblaciones más altas sin mostrar diferencias significativas entre ellos; a partir de la tercera evaluación y hasta el final el glifosato presentó bajas poblaciones de la maleza, situación que es de esperar de acuerdo con el espectro de control de ese herbicida (Vencill 2002). Durante las evaluaciones dos, tres y cuatro los tratamientos de chapea, glufosinato y monitoreado mostraron cantidades similares de la maleza. A partir de la quinta y hasta la novena evaluación el uso de chapea mostró un incremento de las poblaciones de la malezas y junto con el monitoreado presentó para ese momento los valores más altos, bajo las condiciones del agroecosistema seleccionado (Figura 1).

En el manejo de poáceas, Acosta y Agüero (2002 b) encontraron que tratamientos químicos con glifosato, glufosinato y paraquat fueron más eficientes para bajar las poblaciones de $P$. conjugatum y $P$. polygonatum, en tanto que la práctica de la chapea para el control de las mismas fue deficiente ya que mostró densidades de estas poáceas, similares a las del testigo a libre crecimiento.

\section{Axonopus micay}

Todos los tratamientos iniciaron con poblaciones similares de Axonopus micay (Figura 2). No se presentaron diferencias significativas entre tratamientos hasta la novena evaluación. Sin embargo, cabe señalar que con excepción de la tercera y cuarta evaluación, el mayor número de individuos se presentó en los tratamientos con glufosinato, donde se llegó a contabilizar un promedio de 297 individuos $/ \mathrm{m}^{2}$ comparado con glifosato que presentó 45 individuos $/ \mathrm{m}^{2}$ en la novena evaluación.

El incremento en la cantidad de plantas observadas en la cuarta evaluación, puede ser probablemente el resultado de que la chapea no destruye totalmente las estructuras reproductivas sino que favorece una 


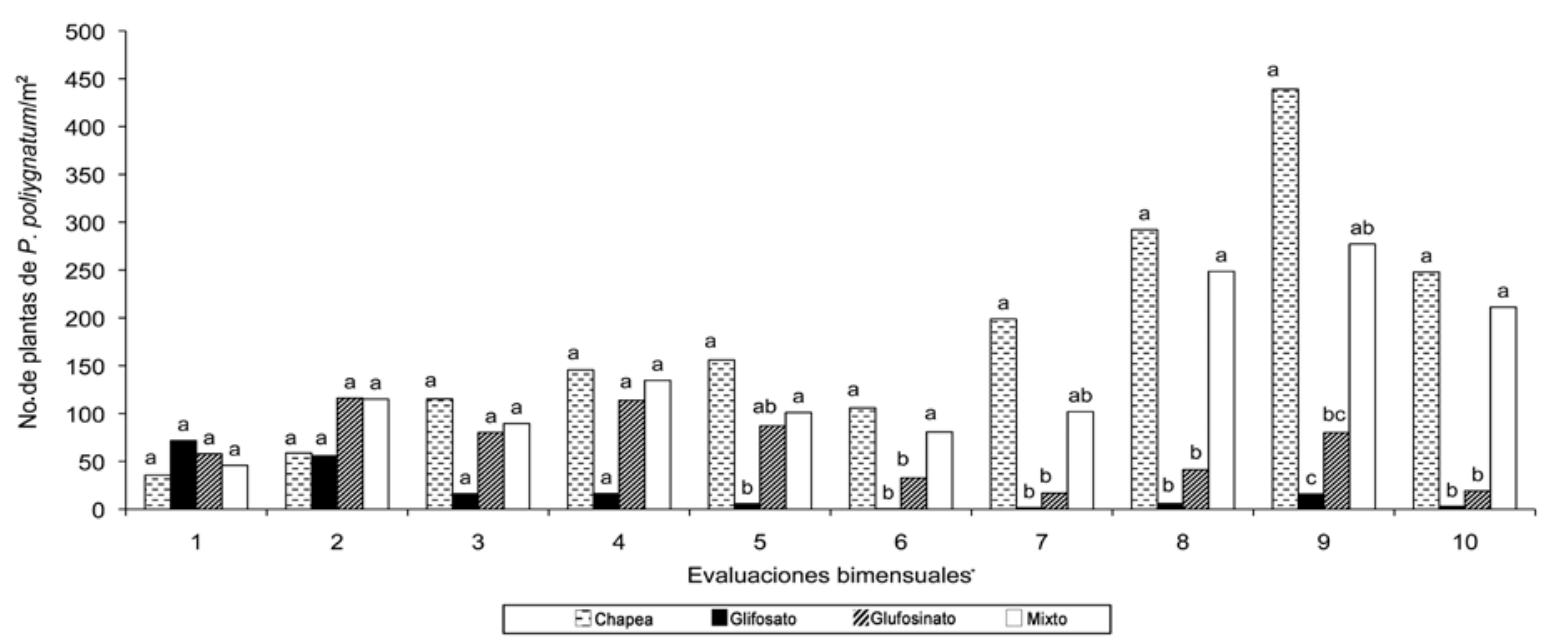

Figura 1. Dinámica poblacional de Panicum polygonatum bajo diferentes tratamientos bimensuales de manejo de malezas en el cultivo del banano. Finca Taciafa, Cariari de Pococí, Limón, Costa Rica, 2005.

* Medias con igual letra en cada barra del gráfico (para cada evaluación) no difieren entre si según la prueba de DMS (P<0,05\%).

dispersión de las mismas y la formación de rebrotes que aumentan el número de individuos.

En tanto que, las diferencias estadísticas observadas en las dos últimas mediciones evidenciaron el poco efecto del glufosinato sobre las poblaciones de esta poácea en comparación con el glifosato; situación que puede estar relacionada con el hecho que el primero de estos productos actúa en forma de contacto (Vencill 2002) y no se transloca en la planta, lo que permite en algunos casos la sobrevivencia de éstas y

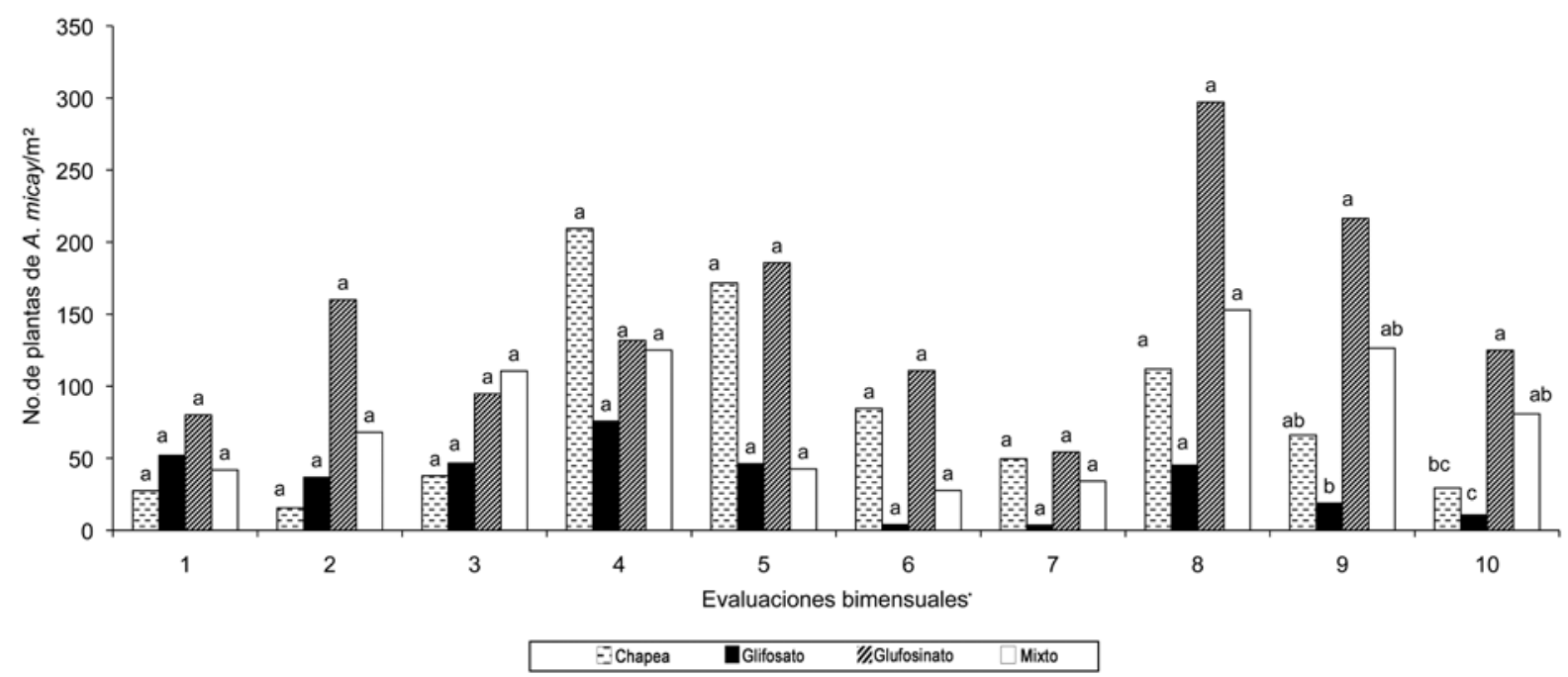

Figura 2. Dinámica poblacional de Axonopus micay bajo diferentes tratamientos bimensuales de manejo de malezas en el cultivo de banano. Finca Taciafa, Cariari de Pococí, Limón, Costa Rica, 2005.

*Medias con igual letra en cada barra del gráfico (para cada evaluación) no difieren entre si según la prueba de DMS (P<0,05\%). 
favorece el aumento de sus individuos, a diferencia del glifosato que puede transportarse por toda la planta (Vencill 2002) y en muchos casos logra eliminarla totalmente (Figura 2).

\section{Comparación entre las poblaciones de las dos prin- cipales especies de poáceas}

A través de cada una de las evaluaciones en los diferentes tratamientos se observó la presencia de cierto grado de interacción entre las dos principales poáceas presentes en el experimento, $P$. polygonatum y A. micay. En el tratamiento con chapea las dos especies comenzaron con valores similares, pero ya para la segunda evaluación $P$. polygonatum presentó un aumento en el número de individuos en comparación con A. micay Las poblaciones de $P$. polygonatum aumentaron en los siguientes conteos con valores que triplicaron los datos iniciales en tanto que A. micay sólo mostró un leve aumento en sus poblaciones en la cuarta evaluación a partir de la cual el número de individuos en este tratamiento tendió a disminuir (Figura 3). P. polygonatum manifestó casi siempre valores más altos que A. micay, posiblemente la presencia en la primera de ellas de una alta cantidad de nudos a lo largo del culmo, como se indicó anteriormente, favorezcan un aumento considerable de sus poblaciones al realizar la chapea.

Las parcelas que llevaban las aplicaciones con glifosato presentaron en un inicio mayor cantidad de P. polygonatum que de A. micay (Figura 4) comportamiento que se mantuvo en la segunda evaluación; con una tendencia a la disminución del número de in-

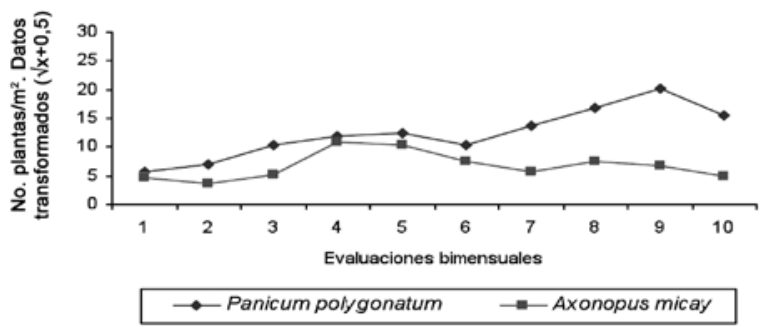

Figura 3. Comportamiento poblacional de $P$. polygonatum y A. micay bajo aplicación bimensual de chapea en el cultivo de banano Finca Taciafa, Cariari de Pococí, Límón, Costa Rica, 2005.

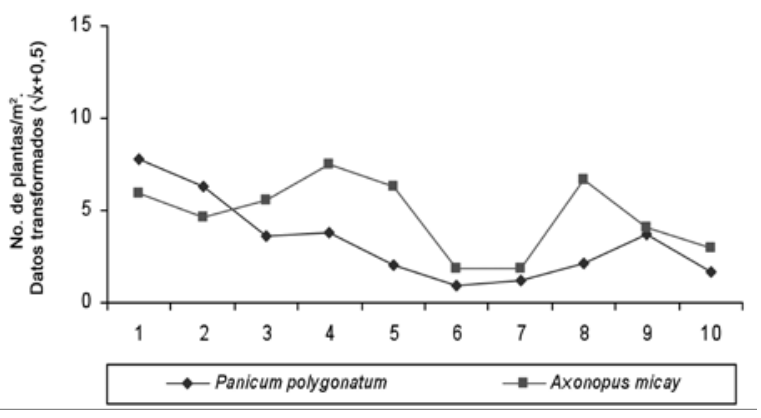

Figura 4. Comportamiento poblacional de P. polygonatum y A. micay bajo aplicación bimensual de glifosato, en el cultivo de banano. Finca Taciafa, Cariari de Pococí, Límón, Costa Rica, 2005.

dividuos de las dos especies. En la tercera evaluación la población de $P$. polygonatum disminuyó y presentó valores menores que la de A. micay; especie que incrementó sus poblaciones, llegando a sus máximos en la cuarta y octava evaluación para luego empezar a disminuir, en tanto que $P$. polygonatum continuó siempre con poblaciones más bajas.

Cabe indicar que el glifosato es un producto para el manejo general de poáceas, observándose sin embargo un mejor control de $P$. polygonatum. Esta situación pareciera estar relacionada con las características anatómicas de ambas especies ya que $P$. paniculatum es más estolonífera que A. micay, la cual aunque también presenta estas estructuras se encuentran en menor cantidad y tiene un crecimiento más cespitoso (Pohl 1980) por lo que posiblemente exista menos translocación del producto a diferentes puntos de la planta que puedan afectarla.

En las áreas tratadas con glufosinato, desde el inicio se presentaron mayores poblaciones de $A$. micay que de $P$. polygonatum, diferencia que tendió a mantenerse e incrementarse durante casi todas las evaluaciones hasta el final del experimento (Figura 5). En ninguna de las dos especies se logró observar una disminución sustancial de sus poblaciones. Como se mencionó anteriormente, el glufosinato es un herbicida de contacto que no se transloca dentro de la planta por lo que no logra llegar a puntos de crecimiento y afectar completamente el desarrollo de algunas de las especies tratadas con el mismo. 


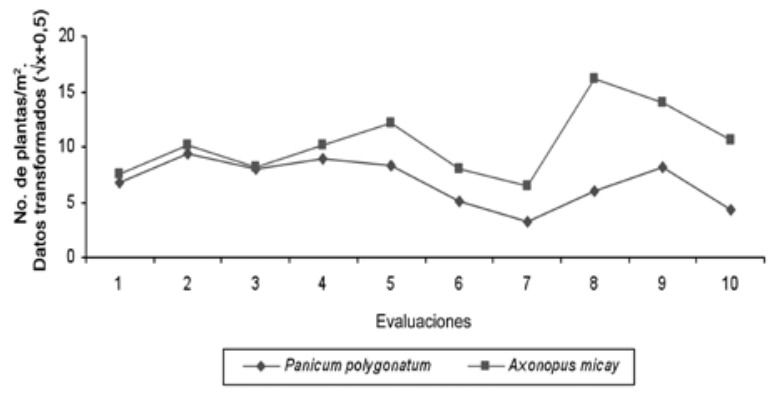

Figura 5. Comportamiento poblacional de P. polygonatum y A. micay bajo aplicación bimensual de glufosinato, en el cultivo del banano. Finca Taciafa, Cariari de Pococí, Limón, Costa Rica. 2005.

\section{AGRADECIMIENTOS}

Al personal técnico y de campo de la Corporación Bananera Nacional (CORBANA), Costa Rica.

\section{LITERATURA CITADA}

Acosta, L; Agüero, R. 2001. Levantamiento taxonómico y densidad de la flora vascular asociada al agroecosistema banano (Musa AAA) en el trópico húmedo de Costa Rica. In XLVII Reunión del Programa Cooperativo Centroamericano para el Mejoramiento de Cultivos y Animales. San José, Costa Rica. 113 p.

Acosta, L; Agüero, R. 2002 a. Banco de semillas de poáceas en un agroecosistema de banano y su relación con el potencial regenerativo. In XV Reunión de ACORBAT. Cartagena, Colombia. Memorias ACORBAT. p. 331335.

Acosta, L; Agüero, R. 2002 b. Combate de Panicum zizanoides H.B.K. y Paspalum conjugatum Beg. en banano (Musa AAA) en el trópico húmedo de Costa Rica. Agronomía Mesoamericana 13(2):117-121.
Acosta, L; Agüero, R. 2002 c. Dinámica de poblaciones de ciperáceas bajo diferentes sistemas de manejo en una plantación de banano en el trópico húmedo de Costa Rica. In XV Reunión de ACORBAT. Cartagena, Colombia. Memorias ACORBAT. p 310-314.

Agüero, R; Rojas, M. 1996. Otras malezas gramíneas. In Agüero, R. eds. Malezas del arroz y su manejo. San José, C.R., I.M.R.SA. p. 99-134.

Gamboa, B. 1983. Combate de malezas en banano en Cariari de Pococí. Tesis presentada para optar al título de Ingeniero Agrónomo. San José, Costa Rica. Facultad de Agronomía. 49 p.

Herrera, W. 1985. Clima de Costa Rica: vegetación y clima de Costa Rica. Vol 2. San José, Costa Rica. Ed. UNED. $118 \mathrm{p}$.

Holdridge, L. 1979. Ecología basada en zonas de vida. San José, Costa Rica. IICA. 216 p.

Krebs, CJ. 1985. Ecology: Experimental analysis of distribution and abundance. Nueva York, Harper \& Row. 694 p.

Pinilla, C; García, J. 2002. Manejo integrado de malezas en plantaciones de banano. Colombia, Uniban S. A. 295 p.

Pitty, A; Muñoz, R. 1993, Guía práctica para el manejo de malezas. Eds. H. Barletta; JM Muñoz. Honduras, DPV-EAP. 223 p.

Pohl, WR. 1980. Flora costaricensis. Family \#15, Gramineae. Ed. W. Burger. USA. Fieldiana Bot., New Ser. 4.608 p.

Rijn, PJ van. 2000. Weed management in the humid and sub-humid tropics. The Netherlands. Royal Tropical Institute. Kit Press. 240 p.

Soto, M; Soto, E; Solís, P; López, A. 1992. Siembra y operaciones del cultivo. In Soto, M. eds. Bananos. Cultivo y comercialización. 2 ed. San José, C.R. Litografía e Imprenta LIL. 674 p

Vencill, WK. (ed). 2002. Herbicide Handbook. WSSA. Kansas, USA.493 p.

Young, J; Evans, R. 1976. Responses of weed populations to humans manipulations of the natural environment. Weed Science 24(2):186-190. 